\title{
An Antioxidant Capacity Assay Using a Polyvinyl Alcohol-Based DPPH Pellet
}

\author{
Yeong Hee Ahn, Jong Shin Yoo, and Sung Ho Kim ${ }^{\dagger}, *$ \\ Mass Spectrometry Division, Korea Basic Science Institute, Cheongwon, Korea \\ ${ }^{\dagger}$ Department of Chemistry, Soonchunhyang University, Asan 336-745, Korea.*E-mail: shkim71@sch.ac.kr \\ Received April 17, 2010, Accepted July 26, 2010
}

\begin{abstract}
To assay for antioxidant capacity of natural products considered important in producing human health benefits, a practical and economical method using pellet techniques was developed. A standard visualizing reagent, 1,1diphenyl2-picryl-hydrazyl (DPPH), was mixed with a water-miscible polyvinyl alcohol (PVA), serving as a solid phase support for the DPPH reagent. A DPPH pellet was prepared by dropping a small volume of the DPPH solution onto PET film, and drying in an oven. The PVA-based DPPH pellet was dissolved into water, in which the water-miscible PVA plays as a non-ionic surfactant to help the DPPH reagent to be dissolved into the solvent. Using the DPPH assay, the antioxidant capacity of water-soluble extracts of black soybean, barley, green tea, and green gram was examined. Among the natural products tested, green tea showed the highest antioxidant capacity. This PVA-based DPPH antioxidant assay can be further applied in the natural food, raw plant material, and health product inspection field.
\end{abstract}

Key Words: Antioxidant assay, DPPH, PVA, Pellet

\section{Introduction}

Although human skin is equipped with a system to defend against potentially harmful reactive oxygen species (ROSs), produced primarily by normal or abnormal aerobic metabolism, breakdown of the skin's defensive system from exposure to ultraviolet light can lead to aging and malignant skin tissues. ${ }^{1}$ ROSs are typically removed from skin cells or directly inactivated by a variety of antioxidants, substances capable of delaying or preventing oxidation of an oxidizable substrate present in cells. Removing ROSs by eating antioxidant-rich foods or applying antioxidant substances directly to the skin via cosmetic lotions can minimize oxidative stress-induced skin damage. ${ }^{2}$

Isoflavones, catechins, and proanthocyanidins are antioxidants commonly extracted from plants, such as black soybean, green tea, herbs, and vegetables. Antioxidants used in food or as cosmetic ingredients can offer health benefits. Depending on a specific antioxidant's properties and the ingestion or application method, different assay methods to measure antioxidant effects have been developed, including the ferric reducing antioxidant power (FRAP) assay, ${ }^{3-5}$ total radical-trapping antioxidant parameter (TRAP) assay, ${ }^{4-6}$ oxygen radical absorbance capacity (ORAC) assay, ${ }^{5,7}$ ABTS (2,2'-azinobis [3ethylbenzothia-

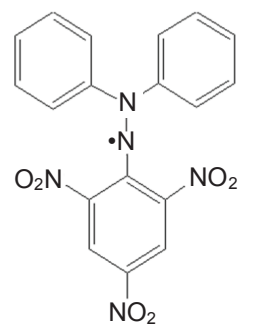

Figure 1. Chemical structure of the DPPH radical. zoline 6-sulfonate]) assay, ${ }^{5}$ and DPPH (1,1-diphenyl-2-picrylhydrazyl) assay.

DPPH is a stable free radical with an unpaired electron at one nitrogen atom on a nitrogen-nitrogen bridge (Fig. 1). Various antioxidant DPPH assay approaches were introduced using high-throughput and on-line high performance liquid chromatography (HPLC) technique, which is especially useful to assay concurrently antioxidant activity of each of interesting multicomponents present in a sample. ${ }^{10,11}$ However these methods require use of expensive analytical facilities like an on- line HLPC system and a specific readout apparatus, which makes a single assay expensive. To evaluate a more practical and economical protocol for rapid assaying overall antioxidant capacity of extracts of a natural product we tested an antioxidant assay method where the visualizing standard reagent DPPH was a solid-state pellet. The pellets were produced by mixing DPPH and a water-miscible polyvinyl alcohol (PVA). After developing an assay using the PVA-based DPPH pellet, the antioxidant capacity of water-soluble extracts of some natural products was examined.

\section{Experiment}

DPPH pellet preparation. A $1 \mathrm{mM}$ DPPH standard solution was prepared by dissolving DPPH (Fluka) in ethyl alcohol (EtOH). DPPH pellets were prepared by mixing equal volume of the DPPH solution and 5\% PVA 500 (Duksan Pure Chemical Co.). A $400 \mu \mathrm{L}$ drop of the solution was placed onto PET film (Fig. 2a), and dried in an oven (J-NDS-1, JISICO) for 30 min at $90{ }^{\circ} \mathrm{C}$. After drying, each DPPH pellet was excised and used in a DPPH antioxidant assay (Fig. 2b).

Standard test solution and calibration curve development. For the DPPH standard solution assay, a stock vitamin C solution (Duksan Pure Chemical Co.) was dissolved into EtOH at the final concentration of $100 \mathrm{ppm}$. The solution was further 


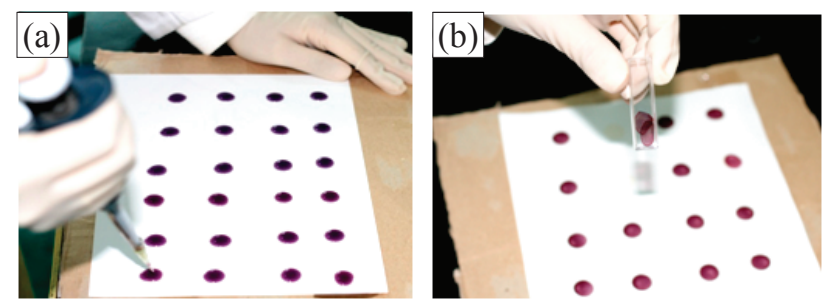

Figure 2. Preparation of PVA-based DPPH pellet: (a) spotting a drop of DPPH solution onto PET film; (b) detaching a dried PVA-based DPPH pellet.

diluted with EtOH into test concentrations of 1, 2, 3, 4, and $5 \mathrm{ppm}$. A portion $(1 \mathrm{~mL})$ of each diluted solution was mixed with an assay solution which was prepared by spiking $200 \mu \mathrm{L}$ DPPH to $1 \mathrm{~mL}$ EtOH. Ultraviolet (UV) radiation absorbance (at $519 \mathrm{~nm}$ wavelength) for each test concentration was recorded triplicate using a UV-VIS spectrometer (MACASYS CORP. OPTIZEN 3220UV).

For the DPPH pellet assay, PVA-based DPPH pellets were dissolved in water $(1.2 \mathrm{~mL})$. After addition of the DPPH solution to the diluted vitamin $\mathrm{C}$ solutions described above, UV absorbance (at $519 \mathrm{~nm}$ ) for each test concentration was recorded replicate.

Measurement of antioxidant capacities of natural products using the DPPH pellet method. Extracts of natural plant products from black soybean, barley, green tea, and green gram (also known as mung bean) were prepared by incubating $2 \mathrm{~g}$ of plant product powder in water for $2 \mathrm{~h}$ at $60{ }^{\circ} \mathrm{C}$, gently rotating the solution in a rotary evaporator, and centrifuging the final product (13,000 rpm, $2 \mathrm{~min})$. The supernatant from each extract was freeze-dried (PVTFD30A, Ilshin Corp.) and stored for later analysis. A portion $(0.1 \mathrm{~g})$ of each dried extracts was dissolved with $\mathrm{EtOH}$ to be $1000 \mathrm{ppm}$ concentration. The final extract solution $(1 \mathrm{~mL})$ was mixed with a PVA-based DPPH pellet dissolved in $1.2 \mathrm{~mL}$ of water. UV absorbance (at $519 \mathrm{~nm}$ ) for each sample was recorded triplicate.

\section{Results and Discussion}

Calibration curves in assay by solution and pellet methods. Because the accuracy of the DPPH assay can be influenced by experimental variables involving radical-scavenging reaction efficiency and sample conditions, such as DPPH concentration, composition of solvents, incubation time, and $\mathrm{pH}$ of the reaction mixture, we first constructed calibration curves using the standard solution and pellet methods. Table 1 shows UV absorbance variations from increasing concentrations of vitamin $\mathrm{C}$ antioxidant solution using the standard DPPH solution. Experimental results were also graphically analyzed (Fig. 3). A high degree of linearity was observed across the range of vitamin $\mathrm{C}$ concentrations. Results obtained using the pellet method (Table 2) also exhibited a high degree of linearity across the range of vitamin $\mathrm{C}$ concentrations (Fig. 4). However, absolute absorbance values measured using the pellet method are significantly different from those of the standard solution method, which is further discussed in the following section.
Table 1. Variation of UV absorbance with concentration of vitamin C using the standard solution method

\begin{tabular}{ccc}
\hline Vitamin C concentration (ppm) & Absorbance & RSD (\%) \\
\hline 0 & 0.897 & 1.8 \\
1 & 0.803 & 2.8 \\
2 & 0.693 & 2.1 \\
3 & 0.604 & 6.6 \\
4 & 0.501 & 3.3 \\
5 & 0.374 & 3.3 \\
\hline
\end{tabular}

Table 2. UV absorbance for increasing vitamin $\mathrm{C}$ concentrations using the pellet-based method

\begin{tabular}{ccc}
\hline Vitamin C concentration (ppm) & Absorbance & RSD (\%) \\
\hline 0 & 0.914 & 3.9 \\
1 & 0.662 & 1.6 \\
2 & 0.555 & 4.8 \\
3 & 0.444 & 6.4 \\
4 & 0.368 & 3.9 \\
5 & 0.277 & 6.6 \\
\hline
\end{tabular}

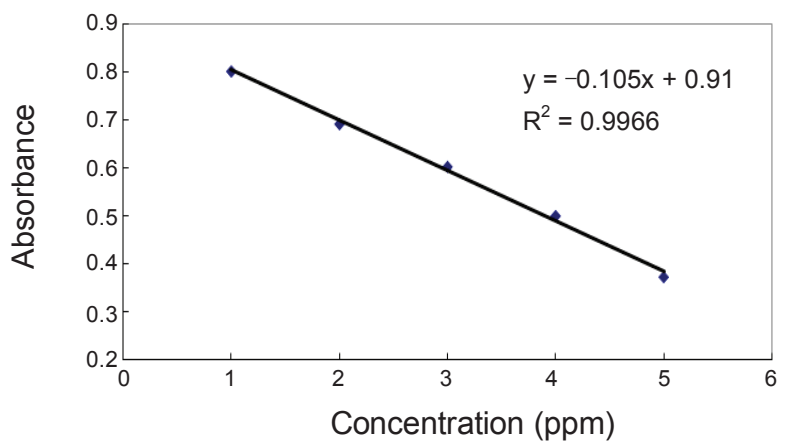

Figure 3. Plot of UV absorbance vs. vitamin C concentration obtained using the standard solution method.

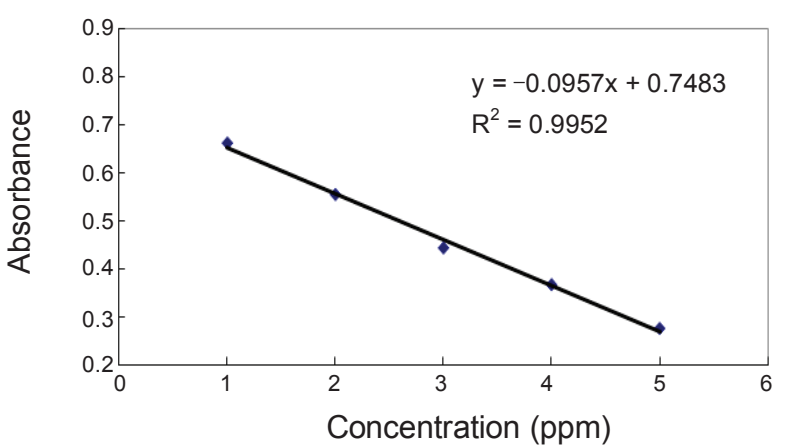

Figure 4. Plot of UV absorbance $v s$. vitamin $\mathrm{C}$ concentration obtained using the pellet-based method.

Validation of the pellet method by calibration curve analysis. Absorbance values of the various vitamin $\mathrm{C}$ concentration solutions obtained from the pellet method were compared with those obtained from the standard solution method. The spectro- 


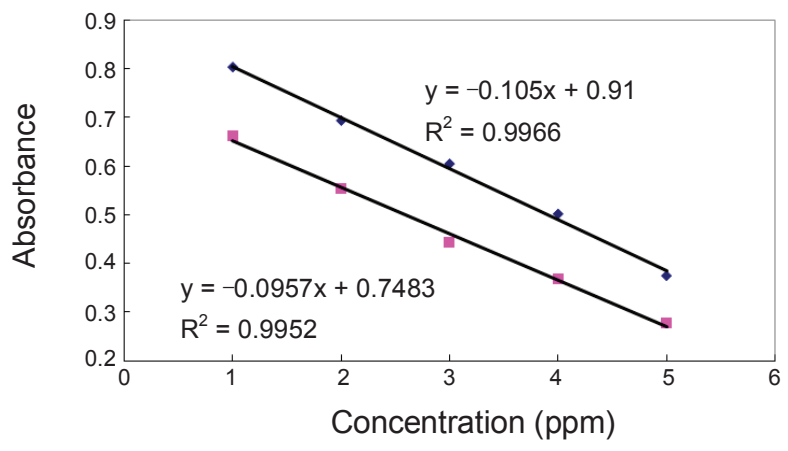

Figure 5. Comparison of UV absorbance variations from increasing vitamin $\mathrm{C}$ concentrations for the two assay methods: represent data for the standard solution method; $\square$ represent data for the pellet-based method.

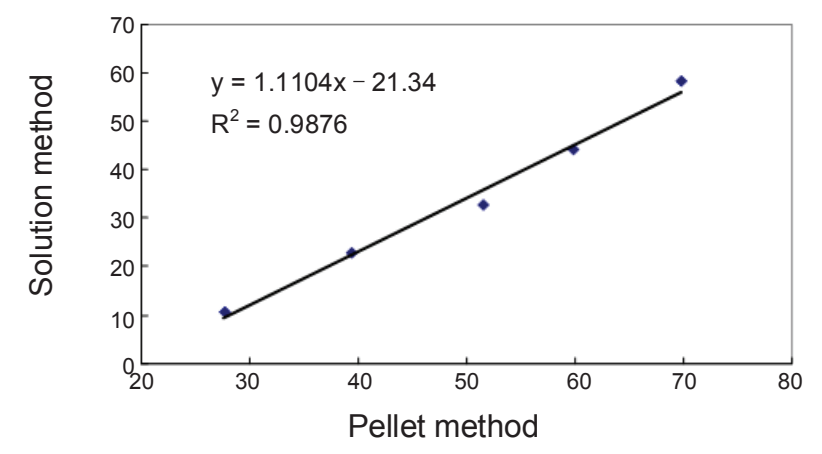

Figure 6. Relationship between $\mathrm{EDA}_{\mathrm{s}}$ and $\mathrm{EDA}_{\mathrm{p}}$ values obtained by the solution and pellet-based methods, respectively.

photometric total absorbance $\left(\mathrm{A}_{\text {total }}\right)$ measurement for a sample can be expressed as:

$$
A_{\text {total }}=A_{f-r}+A_{n-r}+A_{m}
$$

where $\mathrm{A}_{\mathrm{f}-\mathrm{r}}$ represents absorbance by free radicals of $\mathrm{DPPH}$, $A_{n-r}$ represents the non-radical forms of DPPH generated by the addition of an antioxidant solution, and $\mathrm{A}_{\mathrm{m}}$ corresponds to a sum of absorbance factors due to solvent composition and the multiple minor components dissolved in the assay solution (e.g., PVA in the pellet method). We assumed that differences in the concentration terms $\mathrm{A}_{\mathrm{f}-\mathrm{r}}$ and $\mathrm{A}_{\mathrm{n}-\mathrm{r}}$ between the two methods was not significant. However, differences between the concentration term $\mathrm{A}_{\mathrm{m}}$ for the two methods can be significant, because we assumed that $A_{m}$ in the pellet method would be affected by a component (e.g., PVA) added in the pellet preparation process. The presence of PVA dissolved into the sample solution from the pellet can increase the solubility of DPPH reagent owing to the role of PVA as a non-ionic surfactant and thus affect UV absorbance measurements. Additionally, the different solvent compositions used in the two methods can affect absolute absorbance measurements. A solvent composition including water was used for the pellet-based DPPH assay, so it was anticipated that absolute absorbance values obtained using the pellet method would be significantly different from those obtained using the standard solution method (Fig. 5). Proper correction of absor-
Table 3. EDA values of vitamin $\mathrm{C}$ concentrations measured by the standard solution and pellet-based methods

\begin{tabular}{cccccc}
\hline \multirow{2}{*}{$\begin{array}{c}\text { Vitamin C } \\
\text { concentration (ppm) }\end{array}$} & \multicolumn{2}{c}{ Solution method } & & \multicolumn{2}{c}{ Pellet method } \\
\cline { 5 - 6 } \cline { 5 - 6 } & Absorbance & $E^{2} A_{s}(\%)$ & & Absorbance & $E^{2} A_{p}(\%)$ \\
\hline 0 & 0.897 & - & & 0.914 & - \\
2 & 0.803 & 10.48 & & 0.662 & 27.57 \\
3 & 0.693 & 22.74 & & 0.555 & 39.28 \\
4 & 0.604 & 32.66 & & 0.444 & 51.42 \\
5 & 0.501 & 44.15 & & 0.368 & 59.74 \\
\hline
\end{tabular}

Table 4. Corrected EDA values of water-soluble extracts of each plant product measured by the pellet-based method

\begin{tabular}{cccc}
\hline Natural product & Absorbance & RSD $(\%)$ & EDA $_{\mathrm{c}}(\%)$ \\
\hline Black soy-bean & 1.341 & 6.0 & 46.74 \\
Barley & 1.163 & 9.6 & 27.22 \\
Green tea & 2.565 & 7.6 & 73.69 \\
Green gram & 1.188 & 4.4 & 30.03 \\
\hline
\end{tabular}

bance values obtained using the pellet method is necessary to accurately measure the antioxidant capacity of a sample.

Calculation of the electron-donating ability of an antioxidant substance. Electron-donating ability (EDA) of an antioxidant can be calculated from spectrophotometric absorbance value measurements as:

$$
\begin{aligned}
& \operatorname{EDA}(\%)= \\
& \left\{\left(\mathrm{A}_{\text {initial }} \mathrm{DPPH}\right.\right. \\
& \left.\left.-\mathrm{A}_{\text {final DPPH }}\right) / \mathrm{A}_{\text {initial DPPH }}\right\} \times 100
\end{aligned}
$$

where $\mathrm{A}_{\text {initial DPPH }}$ represents the absorbance of DPPH solution with no antioxidant substance present, and $\mathrm{A}_{\text {final DPPH }}$ represents the absorbance of DPPH solution after adding an antioxidant substance. EDA values for each test concentration of vitamin C obtained from each of the two methods differed (Table 3), so it was necessary to apply a correction to the pellet-based EDA $\left(E A_{p}\right)$ values. The relationship between the two data sets is shown in Figure 6, and a corrected value $\left(\mathrm{EDA}_{\mathrm{c}}\right)$ for $\mathrm{EDA}_{\mathrm{p}}$ is calculated as:

$$
\mathrm{EDA}_{\mathrm{c}}=1.11 \times \mathrm{EDA}_{\mathrm{p}}-21.34
$$

Application of the DPPH pellet method for measuring antioxidant capacity. Among the many natural plant products known to have beneficial effects on human health, black soybean, barley, green tea, and green gram were selected for this study to measure their antioxidant capacities using the DPPH pellet method. Water-soluble extract of green tea exhibited the highest antioxidant capacity of the extracts tested (Table 4). Green tea is known to contain high levels of antioxidant components such as catechin (a polyphenolic antioxidant), and has been shown to provide various health benefits, based on extensive studies in humans and animals. ${ }^{12-14}$ The DPPH antioxidant assay using 
PVA-based pellets offers a simple and practical method for measuring the antioxidant capacity of natural products.

\section{Conclusions}

A practical and economical assay method for antioxidants using a PVA-based DPPH pellet was developed. Because the PVA polymer is used as a solid phase support for the DPPH reagent in the pellet and the PVA-based pellet has been preformulated, the assay process could be further simplified. The easy handling and facile nature of the pellet method can play an important role in the rapid assay of a large number of samples. Thus, this method may serve as a useful assay tool in the natural food, raw plant material, and health product inspection field.

\section{References}

1. Boo, Y. C.; Jeon, C. O.; Kim, J. S.; Park, S. N. Preprint, 1 st Scientific Conference of the Asian Soc. Cosm. Sci. 1993, 143-149.

2. Lloyd, B. J.; Siebenmorgen, T. J.; Beers, K. W. Cereal Chem. 2000, $77,551-555$.
3. Benzie, I. F.; Strain, J. J. Anal. Biochem. 1996, 239, 70-76.

4. Koutelidakis, A. E.; Argiri, K.; Serafini, M.; Proestos, C.; Komaitis, M.; Pecorari, M.; Kapsokefalou, M. Nutrition 2009, 25, 453-458.

5. Dudonné, S.; Vitrac, X.; Coutière, P.; Woillez, M.; Mérillon, J. M. J. Agric. Food Chem. 2009, 57, 1768-1774.

6. Ghiselli, A.; Serafini, M.; Natella, F.; Scaccini, C. Free Radic. Biol. Med. 2000, 29, 1106-1114.

7. Prior, R. L.; Hoang, H.; Gu, L.; Wu, X.; Bacchiocca, M.; Howard, L.; Hampsch-Woodill, M.; Huang, D.; Ou, B.; Jacob, R. J. Agric. Food Chem. 2003, 51, 3273-3279.

8. Alma, M. H.; Mavi, A.; Yildirim, A.; Digrak, M.; Hirata, T. Biological and Pharmaceutical Bulletin 2003, 26, 1725-1729.

9. Kordali, S.; Cakir, A.; Mavi, A.; Kilic, H.; Yildirim, A. J. Agric. Food Chem. 2005, 53, 1408-1416.

10. Bandoniene, D.; Murkvic, M. J. Agric. Food Chem. 2002, 50, 2482-2487.

11. Cheng, Z.; Jeffrey, M.; Yu, L. J. Agric. Food Chem. 2006, 54, 74297436.

12. Chyu, K. Y.; Babbidge, S. M.; Zhao, X.; Dandillaya, R.; Rietveld, A. G.; Yano, J.; Dimayuga, P.; Cercek, B. Circulation 2004, 109, 2448-2453.

13. Mittal, A.; Pate, M. S.; Wylie, R. C.; Tollefsbol, T. O.; Katiyar, S. K. Int. J. Oncol. 2004, 24, 703-710.

14. Katiyar, S.; Elmets, C. A.; Katiyar, S. K. J. Nutr. Biochem. 2007, $18,287-296$ 